\title{
Dynamics of a stochastic linear-growth equation with spatiotemporal correlated noise
}

\author{
Ning-Ning Pang \\ Department of Physics, National Taiwan University, Taipei, Taiwan, Republic of China
}

(Received 10 July 1997)

\begin{abstract}
The Edwards-Wilkinson (EW) equation [Proc. R. Soc. London, Ser. A 381, 17 (1982)] with long-range spatiotemporal noise correlation of the power-law form for arbitrary substrate dimensionality is analyzed in detail. We obtain in closed form not only the interfacial width but also various correlation functions characterizing the system. This information can be employed for the comparison with experiments directly, permitting determination of various parameters. Furthermore, we demonstrate explicitly that the interface described by the EW equation with correlated noise for arbitrary substrate dimensionality is still a self-affine fractal governed by dynamic scaling. [S1063-651X(97)07112-2]

PACS number(s): 05.40. $+\mathrm{j}, 81.10 . \mathrm{Aj}, 02.50 .-\mathrm{r}$
\end{abstract}

The kinetic roughening phenomenon of growing interfaces in random media has brought about much interest for its widespread applications in nature [1]. Mathematically, the partial differential equations with stochastic noise components are often used to describe the continuum limit of the evolution of the growing interfaces [1]. One of the bestknown examples is the Edwards-Wilkinson (EW) equation [2], consisting of two terms: the Laplacian component and the white-noise component. The EW equation has been widely accepted as a standard model appropriate to kinetic roughening phenomena in which the competing effects are surface tension relaxation and the incessant peppering of stochastic noise. Recently, it was pointed out $[3,4]$ that the spatiotemporal white-noise assumption for the EW equation is invalid if the substrate dimensionality is higher than two. Moreover, it shows $[3,4]$ that the noise correlation must be less singular than the $\delta$ function in order to obtain the physically reasonable solution. This motivates us to study the EW equation with long-range noise correlation in the higher (than two) substrate dimensionality in order to see how the interfacial behavior varies with the different noise correlation. In addition, it has also been conjectured recently [5] that the EW equation with temporal correlated noise is suitable for describing the interface roughening in driven magnetic systems with quenched disorder, of which the experimental realization is a diluted antiferromagnet in an applied magnetic field. Jost and Usadel [5] conjectured that the random fields at the moving interface play the role of an effective noise. The correlations between the random fields at the interface have been measured numerically [5] to be temporally power-law correlated, $\left|t-t^{\prime}\right|^{-0.8}$. Therefore, a rigorous study of the EW equation with correlated noise is the very next step necessary for establishing the whole theoretical foundation related to the $\mathrm{EW}$ equation.

In this paper we give a comprehensive analysis of the EW equation with long-range spatiotemporal correlated noise of the power-law form for arbitrary substrate dimensionality. We specifically choose the noise correlation to be long-range correlated of the power-law form for two reasons. First, it is pointed out in Ref. [8] that the power-law correlated noise may represent the effect of removing fast degrees of freedom. Second, the results of this paper then can be used to clarify whether the conjecture [5], related to the domain wall roughening driven through a medium with random fields, is correct. The calculations in this work generalize the previous results in Ref. [6], which mainly deals with the EW equation with spatial correlated noise in $1+1$ dimensions. The outline of this paper is as follows. We first review the usual technique of solving the stochastic diffusion-type equations and point out its limitations. Then we study extensively the interfacial width and the various correlation functions. We obtain not only the values of the critical exponents but also the exact expressions of the scaling functions for arbitrary substrate dimensionality and noise correlation of the power-law form. Finally, we discuss the implications of the results obtained and their applications related to experiments.

Here we consider the interfacial profile $h(\mathbf{x}, t)$ in $d+1$ dimensions, governed by the EW equation with spatiotemporal correlated noise:

$$
\partial_{t} h(\mathbf{x}, t)=\nu \nabla^{2} h(\mathbf{x}, t)+\eta(\mathbf{x}, t),
$$

where $h(\mathbf{x}, t)$ is the interface height, $\mathbf{x}$ is a coordinate in the $d$-dimensional substrate, and $\eta(\mathbf{x}, t)$ represents the noise. The noise $\eta(\mathbf{x}, t)$ has zero mean and its correlated variance is given as

$$
\left\langle\eta(\mathbf{x}, t) \eta\left(\mathbf{x}^{\prime}, t^{\prime}\right)\right\rangle=2 D\left|\mathbf{x}-\mathbf{x}^{\prime}\right|^{2 \rho-d}\left|t-t^{\prime}\right|^{2 \theta-1},
$$

with $0<\rho<d / 2$ and $0<\theta<\frac{1}{2}$ to account for the decaying long-distance noise correlation. For the self-containment of the paper, we first briefly review the usual technique of dealing with the stochastic diffusion-type equations, which has been mentioned in the previous work of Ref. [6]. As usual, by applying a Fourier transformation to $\mathbf{k}$ space, we get

$$
\partial_{t} \widetilde{h}(\mathbf{k}, t)=-\nu k^{2} \widetilde{h}(\mathbf{k}, t)+\widetilde{\eta}(\mathbf{k}, t) .
$$

The solution for Eq. (3), under the assumption of the flat initial condition, is easily obtained

$$
\widetilde{h}(\mathbf{k}, t)=e^{-\nu k^{2} t} \int_{0}^{t} e^{\nu k^{2} \tau} \widetilde{\eta}(\mathbf{k}, \tau) d \tau .
$$

Here the correlated variance between noises in $\mathbf{k}$ space is 


$$
\left\langle\widetilde{\eta}(\mathbf{k}, t) \widetilde{\eta}\left(\mathbf{k}^{\prime}, t^{\prime}\right)\right\rangle=2 \widetilde{D_{\rho}}|\mathbf{k}|^{-2 \rho} \delta^{d}\left(\mathbf{k}+\mathbf{k}^{\prime}\right)\left|t-t^{\prime}\right|^{2 \theta-1} .
$$

For $d=1$, the prefactor $\widetilde{D}_{\rho}$ is simply

$$
\widetilde{D}_{\rho}=\frac{D}{\pi} \int_{0}^{\infty} d u u^{2 \rho-1} \cos u=\frac{D}{\pi} \Gamma(2 \rho) \cos (\rho \pi) .
$$

For $d>1$, the prefactor

$$
\begin{aligned}
\widetilde{D}_{\rho}= & D \frac{S_{d-1}}{(2 \pi)^{d}} \int_{0}^{\infty} d u u^{2 \rho-1} \int_{0}^{\pi} d \theta \sin ^{d-2} \theta \exp (i u \cos \theta) \\
= & D \frac{\sqrt{\pi} S_{d-1} \Gamma\left(\frac{d-1}{2}\right)}{(2 \pi)^{d}} \int_{0}^{\infty} d u u^{2 \rho-1}\left(\frac{2}{u}\right)^{(d-2) / 2} \\
& \times J_{(d-2) / 2}(u) \\
= & D \frac{\sqrt{\pi} 2^{2 \rho-1} S_{d-1}}{(2 \pi)^{d}} \frac{\Gamma\left(\frac{d-1}{2}\right) \Gamma(\rho)}{\Gamma\left(\frac{d}{2}-1-\rho\right)}
\end{aligned}
$$

with $S_{d-1}, \Gamma$, and $J_{(d-2) / 2}$ denoting the surface area of a $(d-1)$-dimensional unit sphere, the Gamma function, and the Bessel function of the first kind [7], respectively. Note that $0<\rho<(d+1) / 4$ is required for the convergence of $\widetilde{D_{\rho}}$. Consequently, the application of the Fourier method is restricted to the range in which the value of the noise spatial correlation exponent $\rho$ is between 0 and $(d+1) / 4$. To the author's knowledge, the limitation for applying the Fourier technique has not been discussed in the literature. The above procedure is a direct generalization of the previous results [6] to arbitrary substrate dimensionality.

The interfacial width $W(L, t)$ is defined as

$$
W^{2}(L, t) \equiv L^{-d}\left\langle\int_{0}^{L} d^{d} \mathbf{x}\left[h(\mathbf{x}, t)-h_{L}(t)\right]^{2}\right\rangle,
$$

with $L$ denoting the lateral segment of the surface, $h_{L}(t)$ $\equiv L^{-d} \int_{0}^{L} h(\mathbf{x}, t) d^{d} \mathbf{x}$, and \langle\rangle the statistical average. The notations for the interfacial width are in accordance with the notations in Ref. [6]. Substituting Eqs. (4) and (5) into Eq. (8), we obtain

$$
W^{2}(L, t)=L^{2 \rho+4 \theta+2-d} f\left(\nu t / L^{2}\right) .
$$

Consequently, the steady-state roughness exponent $\chi=\rho$ $+2 \theta+(2-d) / 2$, revealing a spatiotemporal symmetry through the combination $\rho+2 \theta$, while the dynamical exponent $z=2$, independent of the values of the noise correlation exponents $(\rho$ and $\theta)$. In contrast, the Kardar-Parisi-Zhang (KPZ) equation with correlated noise [8,9] does not share the above symmetries, due to the presence of the nonlinear term $(\nabla h)^{2}$ in the KPZ equation.

In Eq. (9), the scaling function

$$
\begin{aligned}
f\left(\nu t / L^{2}\right)= & \frac{\widetilde{D}_{\rho}}{\theta \nu^{2 \theta+1}} \int \frac{d^{d} \mathbf{q}}{q^{2 \rho+4 \theta+2}} \\
& \times\left(1-\prod_{i=1}^{d} \frac{2-2 \cos q_{i}}{q_{i}^{2}}\right) g\left(q^{2} \nu t / L^{2}\right),
\end{aligned}
$$

where the notations are defined as $\mathbf{q} \equiv\left(q_{1}, q_{2}, \ldots, q_{d}\right)$ and $q$ $\equiv|\mathbf{q}|$ and the function $g\left(y=q^{2} \nu t / L^{2}\right)$ has the form

$$
\begin{aligned}
g(y) & =\int_{0}^{y} d u u^{2 \theta} e^{-u}+e^{-2 y} \int_{0}^{y} d u u^{2 \theta} e^{u} \\
& =\gamma(2 \theta+1 ; y)+(-1)^{2 \theta+1} e^{-2 y} \gamma(2 \theta+1 ;-y),
\end{aligned}
$$

with $\gamma(2 \theta+1 ; \pm y)$ denoting the incomplete gamma function [7]. When $t \rightarrow \infty, g\left(q^{2} \nu t / L^{2}\right)$ approaches a constant $\Gamma(2 \theta+1)$. Thus we find the scaling function

$$
\begin{aligned}
\left.f\left(\nu t / L^{2}\right)\right|_{t \rightarrow \infty}= & \frac{\widetilde{D}_{\rho}}{\theta \nu^{2 \theta+1}} \Gamma(2 \theta+1) \int \frac{d^{d} \mathbf{q}}{q^{2 \rho+4 \theta+2}} \\
& \times\left(1-\prod_{i=1}^{d} \frac{2-2 \cos q_{i}}{q_{i}^{2}}\right),
\end{aligned}
$$

which gives us the amplitude prefactor for the steady-state interfacial width. Note that the restriction $(d-2) / 2<\rho+2 \theta$ $<d / 2$ is necessary to avoid the divergence of Eq. (12). The above restriction on the noise correlation in fact implies that the interfacial roughness exponent $\chi[=\rho+2 \theta+(2-d) / 2]$ is positive and less than 1 , namely, $W_{\text {sat }} / L$ vanishes in the thermodynamic limit. Thus the kinetically roughened interfacial profile, described by the Edwards-Wilkinson equation with long-range spatiotemporal correlated noise, is still smooth on macroscopic length scales. In contrast, the Mullins-Wolf-Villain equation with correlated noise [10]

$$
\partial_{t} h(\mathbf{x}, t)=\nu \nabla^{4} h(\mathbf{x}, t)+\eta(\mathbf{x}, t)
$$

behaves anomalously in the sense that the roughness exponent $\chi$ is larger than 1 for $1+1$ and $2+1$ dimensions. Typically, anomalously scaling interfaces suggest multiscaling behaviors of the system [11]. However, a rigorous theoretical treatment is still lacking at the present time.

In the following, we calculate in detail the various correlation functions. The notations for correlation functions are in accordance with the notations in Ref. [10]. We are interested in the height difference correlation function, which is defined as

$$
C_{g}\left(\mathbf{x}, t, t_{0}\right) \equiv\left\langle\overline{\left[h\left(\mathbf{x}_{0}+\mathbf{x}, t_{0}+t\right)-h\left(\mathbf{x}_{0}, t_{0}\right)\right]^{2}}\right\rangle,
$$

with the overbar denoting the spatial average and \langle\rangle the statistical average. Due to the translational invariance of the system, the spatial average and the statistical average are interchangeable. By definition, the equal-time height difference correlation function is then given by

$$
G\left(\mathbf{x}, t_{0}\right) \equiv C_{g}\left(\mathbf{x}, t=0, t_{0}\right)
$$

and the height difference correlation function in the steady state is given by 


$$
C_{s}(\mathbf{x}, t) \equiv \lim _{t_{0} \rightarrow \infty} C_{g}\left(\mathbf{x}, t, t_{0}\right) .
$$

Since, from the experimental point of view, the equal-time correlation function $G\left(\mathbf{x}, t_{0}\right)$ and the saturated correlation function $C_{s}(\mathbf{x}, t)$ are more accessible quantities to be compared with the experimental data, we give a detailed analysis of $G\left(\mathbf{x}, t_{0}\right)$ and $C_{s}(\mathbf{x}, t)$ in the following.

Substituting Eqs. (4) and (5) into Eqs. (14) and (15), we obtain the equal-time height difference correlation function, through some tedious calculation, as

$$
G\left(\mathbf{x}, t_{0}\right) \equiv C_{g}\left(\mathbf{x}, t=0, t_{0}\right)=|\mathbf{x}|^{2 \rho+4 \theta+2-d} f_{1}\left(\nu t_{0} /|\mathbf{x}|^{2}\right),
$$

where the scaling function

$$
\begin{aligned}
f_{1}\left(\nu t_{0} /|\mathbf{x}|^{2}\right)= & \frac{2 \widetilde{D_{\rho}}}{\theta \nu^{2 \theta+1}} \int \frac{d^{d} \mathbf{q}}{q^{2 \rho+4 \theta+2}} \\
& \times\left(1-e^{i q \cos \alpha}\right) g\left(q^{2} \nu t_{0} /|\mathbf{x}|^{2}\right),
\end{aligned}
$$

with $\alpha$ denoting the angle between $(\mathbf{q}, \mathbf{x})$ and the function $g$ given in Eq. (11). Our calculation explicitly shows that the correlation function does satisfy the usual dynamic scaling hypothesis with the roughness exponent $\chi=\rho+2 \theta+(2$ $-d) / 2$ and the dynamic exponent $z=2$.

When $t_{0} \gg|\mathbf{x}|^{2} / \nu$,

$$
\begin{aligned}
\left.f_{1}\left(\nu t_{0} /|\mathbf{x}|^{2}\right)\right|_{t_{0} \gg|\mathbf{x}|^{2} / \nu}= & \frac{2 \widetilde{D}_{\rho}}{\theta \nu^{2 \theta+1}} \Gamma(2 \theta+1) \int \frac{d^{d} \mathbf{q}}{q^{2 \rho+4 \theta+2}} \\
& \times\left(1-e^{i q \cos \alpha}\right) .
\end{aligned}
$$

Since $t_{0}$ describes how far the system is from the initial state, this information tells us the amplitude of surface fluctuation and the characteristic relaxation time growing with the lateral distance as $|\mathbf{x}|^{\chi}$ and $|\mathbf{x}|^{z}$, respectively. Note that it requires $(d-2) / 2<\rho+2 \theta<d / 2$ for the convergence of Eq. (19). This restriction on the noise correlation is consistent with the one derived in for the convergence of interfacial width. Through the analysis of the interfacial width and the equal-time correlation function, we obtain the same conclusion: $0<\chi<1$.

For $d=1$, we can integrate Eq. (19) as

$$
\begin{aligned}
& \left.f_{1}\left(\nu t_{0} /|\mathbf{x}|^{2}\right)\right|_{t_{0} \gg|\mathbf{x}|^{2} / \nu} \\
& =\frac{4 \bar{D}_{\rho}}{\theta \nu^{2 \theta+1}} \Gamma(2 \theta+1) \int_{0}^{\infty} \frac{d q}{q^{2 \rho+4 \theta+2}}(1-\cos q) \\
& =-\frac{4 \widetilde{D}_{\rho}}{\theta \nu^{2 \theta+1}} \Gamma(2 \theta+1) \Gamma(-2 \rho-4 \theta-1) \\
& \quad \times \cos [(\rho+2 \theta+1 / 2) \pi]
\end{aligned}
$$

for $d>1$,

$$
\begin{aligned}
\left.f_{1}\left(\nu t_{0} /|\mathbf{x}|^{2}\right)\right|_{t_{0} \gg|\mathbf{x}|^{2 / \nu}} & \\
= & \frac{2 \widetilde{D}_{\rho}}{\theta \nu^{2 \theta+1}} \sqrt{\pi} S_{d-1} \Gamma(2 \theta+1) \Gamma\left(\frac{d-1}{2}\right) \int_{0}^{\infty} \frac{d q}{q^{2 \rho+4 \theta+3-d}} \\
& \times\left[\frac{1}{\Gamma\left(\frac{d}{2}\right)}-\left(\frac{2}{q}\right)^{(d-2) / 2} J_{(d-2) / 2}(q)\right] \\
= & -\frac{\widetilde{D}_{\rho}}{\theta \nu^{2 \theta+1}} 2^{d-2-2 \rho-4 \theta} \sqrt{\pi} S_{d-1} \Gamma(2 \theta+1) \Gamma\left(\frac{d-1}{2}\right) \\
& \times \frac{\Gamma\left(\frac{d}{2}-1-\rho-2 \theta\right)}{\Gamma(1+\rho+2 \theta)},
\end{aligned}
$$

with $S_{d-1}, \Gamma$, and $J_{(d-2) / 2}$ denoting the surface area of a $(d-1)$-dimensional unit sphere, the Gamma function, and the Bessel function of the first kind [7], respectively. Consequently, we obtain the asymptotic behavior of the scaling function, which gives us the amplitude prefactor for the equal-time height difference correlation function in the steady state. This information can be used to determine various phenomenological parameters through comparisons with numerical simulations or experimental results.

Next we study $C_{s}(\mathbf{x}, t)$, the height difference correlation function in the steady state. This quantity is really what the usual dynamic renormalization-group analysis [8] refers to. Substituting Eqs. (4) and (5) into Eqs. (14) and (16), we obtain, after straightforward but very tedious calculations,

$$
\begin{aligned}
C_{s}(\mathbf{x}, t) \equiv & \lim _{t_{0} \rightarrow \infty} C_{g}\left(\mathbf{x}, t, t_{0}\right) \\
= & \frac{2 \widetilde{D_{\rho}}}{\theta \nu^{2 \theta+1}} \Gamma(2 \theta+1) \int \frac{d^{d} \mathbf{k}}{k^{2 \rho+4 \theta+2}} \\
& \times\left[1-e^{i \mathbf{k} \cdot \mathbf{x}} \cosh \left(\nu k^{2} t\right)+\frac{e^{i \mathbf{k} \cdot \mathbf{x}}}{\Gamma(2 \theta+1)}\right. \\
& \left.\times \int_{0}^{\nu k^{2} t} d u u^{2 \theta} \cosh \left(\nu k^{2} t-u\right)\right] .
\end{aligned}
$$

By using the simple technique of change of variables, we can rewrite Eq. (22) as

$$
C_{s}(\mathbf{x}, t)=|\mathbf{x}|^{2 \rho+4 \theta+2-d} f_{2}\left(\nu t /|\mathbf{x}|^{2}\right),
$$

where the scaling function

$$
\begin{aligned}
f_{2}\left(\nu t /|\mathbf{x}|^{2}\right)= & \frac{2 \widetilde{D_{\rho}}}{\theta \nu^{2 \theta+1}} \Gamma(2 \theta+1) \int \frac{d^{d} \mathbf{q}}{q^{2 \rho+4 \theta+2}} \\
& \times\left[1-e^{i q \cos \alpha} \hat{g}\left(q^{2} \nu t /|\mathbf{x}|^{2}\right)\right],
\end{aligned}
$$

with $\alpha$ denoting the angle between $(\mathbf{q}, \mathbf{x})$ and the function $\hat{g}\left(y=q^{2} \nu t /|\mathbf{x}|^{2}\right)$ having the form

$$
\hat{g}(y)=\cosh y-\frac{1}{\Gamma(2 \theta+1)} \int_{0}^{y} d u u^{2 \theta} \cosh (y-u) .
$$


Through the studies of $G\left(\mathbf{x}, t_{0}\right)$ and $C_{s}(\mathbf{x}, t)$, we explicitly demonstrate that the spatiotemporal correlations of surface fluctuations are invariant under the rescaling $\mathbf{x} \rightarrow b \mathbf{x}, t \rightarrow b^{z} t$, and $h \rightarrow b^{\chi} h$, regardless of whether the system is in the transient regime or in the steady state. So the system described by the EW equation with long-range spatiotemporal correlated noise is indeed a perfect example affirming the dynamic scaling hypothesis.

Furthermore, motivated by the practical interest in deposition techniques such as molecular-beam epitaxy (MBE), various stochastic differential equations and cellular automata [1] have been proposed recently to describe surface growth via MBE in different environments. Our results regarding correlation functions then can be used to extract various phenomenological parameters, e.g., $\rho, \theta$, and $D / \nu^{2 \theta+1}$, through comparisons with discrete growth models or experiments such as scanning tunneling microscopy (STM) data or diffuse x-ray reflectivity measurements. For example, the noise correlation exponents $(\rho$ and $\theta$ ) either are given in discrete growth models or can be obtained by experimental measurement of disorder correlation. Then the measurement of the equal-time height difference correlation functions of self-affine surfaces in the steady state provides us the information to determine the value of $D / \nu^{2 \theta+1}$, provided the noise correlation exponents are known.

On the other hand, it is conjectured [5] that the EW equation with temporal correlated noise is suitable for describing the interface roughening, above the depinning transition, in driven magnetic systems with quenched random fields, of which the experimental realization is diluted antiferromagnets in applied magnetic fields. The correlations between the random fields at the interface, playing the role of an effective noise, have been measured numerically [5] to be temporally power-law correlated $\left|t-t^{\prime}\right|^{-0.8}$. Thus our results are also useful to prove or disprove the above conjecture through a comparison with data generated from numerical models, e.g., a comparison of the asymptotic functional forms of the correlation functions. The details will be discussed elsewhere [12].

In conclusion, we analyzed in detail the EdwardsWilkinson equation with long-range spatiotemporal noise correlation of the power-law form for arbitrary substrate dimensionality. We obtained in closed form not only the interfacial width but also various correlation functions characterizing the system. This information can be employed for the comparison with experiments directly, permitting determination of various parameters. Furthermore, we demonstrated explicitly that the interface described by the EW equation with correlated noise for arbitrary substrate dimensionality is still a self-affine fractal governed by dynamic scaling and it reveals a spatiotemporal symmetry through the combination $\rho+2 \theta$. Definitely, the most valuable problem is to obtain the full probability functional for the interfacial profile. However, the derivation of the temporal evolution of the full probability functional for the interfacial profile is much more technically challenging since the noise here is long-range spatiotemporal correlated. Work on this problem is planned for the near future.

This work was supported in part by the National Science Council of Republic of China under Grant No. NSC 872112-M-002-019.
[1] See, for example, A.-L. Barabási and H. E. Stanley, Fractal Concepts in Surface Growth (Cambridge University Press, Cambridge, 1995); T. Halpin-Healy and Y.-C. Zhang, Phys. Rep. 254, 215 (1995); J. Krug, Adv. Phys. 46, 139 (1997).

[2] S. F. Edwards and D. R. Wilkinson, Proc. R. Soc. London, Ser. A 381, 17 (1982).

[3] K. Honda, Phys. Rev. E 55, R1235 (1997).

[4] K. Honda, Fractals 4, 331 (1996).

[5] M. Jost and K. D. Usadel, Phys. Rev. B 54, 9314 (1996).

[6] Y.-K. Yu, N.-N. Pang, and T. Halpin-Healy, Phys. Rev. E 50, 5111 (1994).

[7] See, for example, An Atlas of Functions, edited by J. Spanier and K. B. Oldham (Springer-Verlag, Berlin, 1987); Integrals and Series, edited by A. P. Prudnikov, Y. A. Brychkov, and O. I. Marichev (Gordon and Breach, New York, 1986).

[8] E. Medina, T. Hwa, M. Kardar, and Y.-C. Zhang, Phys. Rev. A 39, 3053 (1989).

[9] N.-N. Pang, Y.-K. Yu, and T. Halpin-Healy, Phys. Rev. E 52, 3224 (1995); J. G. Amar, P.-M. Lam, and F. Family, Phys. Rev. A 43, 4548 (1991); C.-K. Peng, S. Havlin, M. Schwartz, and H. Stanley, ibid. 44, R2239 (1991).

[10] S. Majaniemi, T. Ala-Nissila, and J. Krug, Phys. Rev. B 53, 8071 (1996).

[11] S. Das Sarma, S. V. Ghaisas, and J. M. Kim, Phys. Rev. E 49, 122 (1994).

[12] N.-N. Pang (unpublished). 